\title{
Schulpflicht für alle - Zweck oder Mittel? \\ Der verfassungsrechtliche Rahmen für schulische Bildung und Chancen(un)gleichheit vor und nach der Pandemie
}

\section{Zusammenfassung}

Verfassungsrechtlich werden Bildung und Chancengleichheit in Deutschland von der Schulpflicht her gedacht, die wiederum als Ausdruck des staatlichen Bildungs-, Erziehungs- und Integrationsauftrags verstanden und bislang weitgehend mit einer Schulbesuchspflicht gleichgesetzt wird. Die in der Pandemie gesammelten Erfahrungen erschüttern diese Sichtweise und fordern zu einer Neubewertung der verfassungsrechtlichen Grundlage staatlichen Schulehaltens heraus. Sie ergibt, dass sich Schule rechtlich nicht mehr sinnvoll von der Schulpflicht, sondern vom Recht auf Bildung und einer (bedingten) Pflicht zu Bildung her denken lässt.

Schlüsselwörter: Bildungs- und Erziehungsauftrag, Corona-Pandemie, Distanzlernen, Freilernen, Grundrechte, Homeschooling, Schulpflicht, Verfassungsrecht

\section{Compulsory School Attendance: An End in Itself - or a Means to an End?}

How Constitutional Law Frames Education and Equal Opportunities Before and After the Pandemic

\begin{abstract}
In Germany, the concept of compulsory school attendance usually serves as the starting point for constitutional law reasoning about education and equal opportunities. School attendance duty is normally thought of as a corollary of the educational mission of the nation state. The experiences gathered during the Corona pandemic (such as distance learning) have shaken this conventional wisdom and should lead to an unbiased interpretation of the constitutional bases of state schooling. The article argues that, rather than compulsory schooling, the right to education and a (limited) duty to educate oneself is at the heart of the constitutional law framework for schooling.

Keywords: civil liberties, constitutional law, Corona pandemic, educational mission, compulsory school attendance, free learning, homeschooling
\end{abstract}




\section{Einführung}

Die Schulfrage

„gehört zu den großen Themen in jedem modernen Gemeinwesen. Denn in der Schule gilt es, das Verhältnis von individueller Freiheit und sozialer Einbindung praktisch zu verfertigen - und damit den Bürgerstatus und die öffentliche Ordnung insgesamt zu prägen“ (Wißmann, 2012, S. 17).

Verfassungsrechtlich spitzt sich die Schulfrage am deutlichsten in der Schulpflichtfrage, und d.h. vor allem in diesen Fragen zu: Wer ist ab wann für wie lange unter welchen Bedingungen verpflichtet, die (welche?) Schule zu besuchen? Denn wenn auch das staatliche Schulehalten, der Idee nach egalitäres Angebot von Bildung, Ausbildung und Erziehung, primär ein Akt der Leistungsverwaltung ist, so vertraut der Staat nicht allein auf dessen Attraktivität und freiwillige Inanspruchnahme, sondern sichert diese durch ein dichtgewobenes Netz von Vorschriften. Sie wurzeln im Verfassungsrecht, denn hinter der Schulpflicht kann sich ein intensiver Eingriff in ein Bündel von Grundrechten verbergen. Auch für diejenigen, die Schule nicht als Grundrechtseingriff empfinden, wirkt sie sich als erhebliches Opfer an oder Investition von Lebenszeit sowie emotionaler und intellektueller Energie aus. Ihre besondere Relevanz, möglicherweise ihre besondere Rechtfertigung, jedenfalls aber ihre besondere Schärfe erhält die Schulpflicht dadurch, dass sie als Allgemeine, d.h. unterschiedslos Greifende ausgestaltet und in der deutschen Rechtswirklichkeit in der Tat kaum Ausnahmen zugänglich ist, was mit der Formel „absolute Schulpflicht“ auf den Begriff gebracht werden soll (Reimer, 2008, S. 720).

War die Schulpflicht in den ersten Jahrzehnten der Bundesrepublik juristisch wenig umkämpft, so ist sie - möglicherweise mit einer Zunahme gesellschaftlicher Pluralität (Hanschmann, 2017, S. 103 ff.) - immer mehr herausgefordert. Der folgende Beitrag fragt vor diesem Hintergrund (und unter Ausklammerung des Problems des Absentismus) nach verfassungsrechtlichen Gründen für die und Grenzen der Schulpflicht, und zwar in drei Schritten: in einem Rückblick auf die traditionelle Sichtweise (Kap. 2), in einer kurzen Analyse der schulverfassungsrechtlichen Relevanz der Corona-Pandemie (Kap. 3) und in einem Vorschlag zur Zusammenführung der auseinandergehenden Erfahrungen (Kap. 4).

\section{Schulpflicht in Deutschland: die bisherige Perspektive}

\subsection{Stationen auf dem Weg zur allgemeinen Schulpflicht}

Die Schulpflicht in Deutschland (vor einer näheren Betrachtung hier vorerst als eindeutiges, gewissermaßen monolithisches Phänomen zugrunde gelegt) hat sich in ei- 
nem langen, nicht auf eine kurze Formel zu bringenden Prozess entwickelt (vgl. zum Folgenden Wißmann, 2012, S. 18 ff.), dessen Ausgangspunkt häufig in der Reformation gesehen wird. Zielte die Kirchenreform unter anderem auf den selbständigen Zugang der Christgläubigen zur Heiligen Schrift, so konnte dies auf die Dauer nicht ohne Folgen für das Bildungssystem bleiben und war zugleich eine Herausforderung, derer sich die Gegenreformation annahm. Und bereits Luther selbst brachte 1530 das Zwangsmoment deutlich zum Ausdruck, wenn er in einer Predigt davon sprach, „daß auch die Obrigkeit hier schuldig sei, die Untertanen zu zwingen, ihre Kinder zur Schulen zu halten“ (zit. n. ebd., S. 19). Die sich entwickelnden Territorialstaaten nahmen diesen Auftrag auf, wohl auch aus ökonomischen Interessen. Als Stationen auf dem Weg zu einer Kinder und Jugendliche beiderlei Geschlechts adressierenden, aber auch Eltern und Gesellschaft in Dienst nehmenden und dabei fast ausnahmslos befolgten Pflicht zum Schulbesuch werden beispielsweise das Preußische Schulpflichtedikt von 1717 und das Generallandschulreglement von 1763 (näher Mors, 1986, S. $144 \mathrm{ff}$.) hervorgehoben. Letzteres erlaubte „wohlhabenden Eltern“ allerdings „für ihr Haus und Kinder Privat-Informatores zu halten“ (Wißmann, 2012, S. 23). Ähnliches galt unter dem Preußischen Allgemeinen Landrecht von 1794 (12 II $\$ \$ 43 \mathrm{f}$.), das je nach Perspektive - eine Unterrichtspflicht oder eine bedingte Schulpflicht vorsah:

„\$43. Jeder Einwohner, welcher den nöthigen Unterricht für seine Kinder in seinem Hause nicht besorgen kann, oder will, ist schuldig, dieselben nach zurückgelegtem Fünften Jahre zur Schule zu schicken.

$\S 44$. Nur unter Genehmigung der Obrigkeit, und des geistlichen Schulvorstehers, kann ein Kind länger von der Schule zurückgehalten, oder der Schulunterricht desselben, wegen vorkommender Hindernisse, für einige Zeit ausgesetzt werden" (ebd.).

Die preußische Verfassung von 1850 schrieb in Art. 21 eine Bildungs- und Unterrichtspflicht vor:

„(1) Für die Bildung der Jugend soll durch öffentliche Schulen genügend gesorgt werden.

(2) Eltern und deren Stellvertreter dürfen ihre Kinder oder Pflegebefohlenen nicht ohne den Unterricht lassen, welcher für die öffentlichen Volksschulen vorgeschrieben ist" (ebd.).

Etwas Anderes als die in ihrer Entwicklung hier nur skizzierte Rechtslage ist (selbstverständlich) stets die Rechtspraxis. So geht die Forschung für das 19. und frühe 20. Jahrhundert von erheblichen Umsetzungsdefiziten aus. Jedenfalls werden die Schulbesuchsquoten für Preußen für das Jahr 1816 mit 60 Prozent, für 1871 mit 82 Prozent und für 1911 mit 88 Prozent angegeben (Wißmann, 2012, S. 31 m.w. N.).

Die Weimarer Verfassung (WRV) brachte in Schulfragen, verfassungsrechtlich gesehen, eine Zentralisierung (d.h. die Regelung auf Reichsebene) und eine Etatisierung. 
Art. 145 WRV bekräftigte die allgemeine Schulpflicht, machte sie aber immer noch nicht zu einer ausnahmslosen Pflicht: „Es besteht eine allgemeine Schulpflicht. Ihrer Erfüllung dient grundsätzlich die Volksschule mit mindestens acht Schuljahren und die anschließende Fortbildungsschule bis zum vollendeten achtzehnten Lebensjahr [...].“ Damit blieben Ausnahmen von der Schulbesuchspflicht verfassungsrechtlich möglich und waren auch einfachgesetzlich vorgesehen (Anschütz, 1933, S. 674). Dennoch statuierte der berühmte Kommentator der Weimarer Verfassung mit einer vielzitierten Formel: „Schulpflicht ist Schulbesuchspflicht“ (Anschütz, a.a.O.). Nicht einmal das Reichsschulpflichtgesetz vom 06.07.1938 (RGBl. I, S. 799) machte die Schulpflicht zu einer ausnahmslosen Schulbesuchspflicht (vgl. $\$ 5$ Abs. 1: „Zum Besuch der Volksschule sind alle Kinder verpflichtet, soweit nicht für ihre Erziehung und Unterweisung in anderer Weise ausreichend gesorgt ist."). Statistische Daten zu Ausnahmen von der Schulbesuchspflicht im Nationalsozialismus liegen, soweit ersichtlich, nicht vor (Wißmann, 2012, S. 44). Festhalten lässt sich jedenfalls, dass die allgemeine Schulpflicht immer stärker umgesetzt wurde, dabei aber ihrem Anspruch nach - wie allgemeine Wehrpflicht und andere staatsbürgerliche Pflichten - nie eine ausnahms- und unterschiedslos geltende Pflicht war.

Zu einer Verschärfung der Regelungen kam es, wenn überhaupt, erst nach dem Zweiten Weltkrieg in den Landesverfassungen (bspw. Art. 56 der Hessischen Verfassung vom 01.12.1946, Art. 129 der bayerischen Verfassung vom 02.12.1946); hier wurde die Schulpflicht ohne explizite Ausnahmen (Hessen) oder die Schulpflicht als Schulbesuchspflicht (Bayern) statuiert.

\subsection{Die Schulpflicht unter dem Grundgesetz}

Trotz der eingehenden Regelungen der Landesverfassungen zu Schulangelegenheiten (und der fehlenden Kompetenz des Bundes zur Schulgesetzgebung) rekurrieren Gerichte und Schrifttum für die Klärung schulverfassungsrechtlicher Fragen stets auf Art. 7 Abs. 1 GG. Die lakonische Norm lautet: „Das gesamte Schulwesen steht unter der Aufsicht des Staates." Dabei wird der Aufsichtsbegriff weit verstanden, sodass die Norm nicht nur ein Kontrollrecht, sondern ein Vollrecht enthalte, Sitz der staatlichen Schulhoheit sei. In der Folge verortet die herrschende Meinung auch den (in seinem Kern durchaus unstreitigen) staatlichen Bildungs- und Erziehungsauftrag in Art. 7 Abs. 1 GG. Er dient der Vermittlung von Wissen und Fertigkeiten, die die Schülerinnen und Schüler im weiteren Leben individuell und als Teil der Gesellschaft brauchen. Das Bundesverfassungsgericht hat in einer vielzitierten Entscheidung, die einer bibeltreuen Familie mit grundschulpflichtigen Kindern das Homeschooling versagte, den staatlichen Erziehungsauftrag dahingehend zusammengefasst, er richte

„sich nicht nur auf die Vermittlung von Wissen, sondern auch auf die Heranbildung verantwortlicher Staatsbürger, die gleichberechtigt und dem Ganzen 
gegenüber verantwortungsbewusst an den demokratischen Prozessen in einer pluralistischen Gesellschaft sollen teilhaben können“ (BVerfG, Beschluss der 2. Kammer des Ersten Senats vom 29.04.2003, abgedruckt in: Neue Zeitschrift für Verwaltungsrecht 2003, S. 1113).

In der juristischen Literatur wird der Bildungs- und Erziehungsanspruch des Staates mit dem Selbstentfaltungsrecht der Schülerinnen und Schüler, mit ihrer Berufsfreiheit, aber auch mit der Funktion der Schule als Keimzelle des demokratischen Gemeinwesens begründet (Rux, 2018, S. 40 ff.). Zum Teil wird auf das „Recht auf Bildung sowie auf diskriminierungsfreie Partizipation am Bildungswesen“ rekurriert (Hanschmann, 2017, S. 365). Aus erziehungswissenschaftlicher Perspektive ist das die Schulpflicht rechtfertigende Bildungsversprechen "gerade auch darauf bezogen, sich frei entfalten, sich individuieren zu können, und das auch gegen einen Mainstream oder die ,herrschende Meinung““ (Wiezorek, 2021, S. 27).

Unabhängig von den konkreten Begründungswegen wird das staatliche Bildungsund Erziehungsmandat wiederum als verfassungsrechtliche Begründung der Schulpflicht aufgefasst; und diese wird mehr denn je als Schulbesuchspflicht verstanden - und zwar auch dort, wo der Text der jeweiligen Landesverfassung für ein solches Verständnis keinen Anknüpfungspunkt bietet. Das Recht auf Bildung - ausdrücklich in zahlreichen Landesverfassungen, in Art. 2 des ersten Zusatzprotokolls zur Europäischen Menschenrechtskonvention sowie in Art. 28 der UN-Kinderrechtskonvention verankert - tritt bisher kaum aus den Kulissen. Jedenfalls in den Konfliktfällen spielt es (wohl auch mangels expliziter Verankerung im Grundgesetz) keine nennenswerte Rolle. Vielmehr stehen sich in den Gerichtsentscheidungen verfassungsrechtlich der staatliche Bildungs- und Erziehungsauftrag und die Grundrechte der Schülerinnen und Schüler (insbesondere deren allgemeines Persönlichkeitsrecht aus Art. 2 Abs. 1 i. V.m. Art. 1 Abs. 1 GG) sowie die der Eltern (insbesondere das Elternrecht aus Art. 6 Abs. 2 Satz 1 GG) gegenüber. Bisher galt dabei das Abwägungsmodell, nach dem insbesondere das Elternrecht nicht am Schultor endete, sondern auch in schulbezogenen Fragen fortwirkte. Dem wird in jüngerer Zeit aus der juristischen Literatur heraus ein Abschichtungsmodell entgegengehalten, das die Sphären des Elternrechts einerseits und der staatlichen Schulhoheit andererseits verfassungsrechtlich zu trennen versucht (vgl. hierzu Hanschmann, 2017, S. 101 ff.; Huster, 2013).

Von großer Bedeutung ist nun aber, dass neben den Bildungs- und Erziehungsauftrag in den letzten Jahren sowohl in der allgemeinen Wahrnehmung als auch in der Rechtsprechung als dritte Säule ein Integrationsauftrag der Schule getreten ist. Diese Verselbstständigung verwundert angesichts der erziehungswissenschaftlichen Überlegung, dass "gesellschaftliche Integration gerade über Erziehung und Bildung zu gewährleisten sei“ (Wiezorek \& Reimer, 2021, S. 28). Demgegenüber hat das Integrationsargument mit dem Eigengewicht auch eine beträchtliche Eigendynamik erhalten. So stützte im bereits zitierten Fall zum Homeschooling, in dem eine Familie ihre 
grundschulpflichtigen Kinder aus religiösen Gründen daheim unterrichten wollte, das Bundesverfassungsgericht seine ablehnende Entscheidung auf folgende (seither von anderen Gerichten häufig aufgegriffene) Begründung:

„Die Allgemeinheit hat ein berechtigtes Interesse daran, der Entstehung von religiös oder weltanschaulich motivierten ,Parallelgesellschaften' entgegenzuwirken und Minderheiten auf diesem Gebiet zu integrieren. Integration setzt dabei nicht nur voraus, dass die Mehrheit der Bevölkerung religiöse oder weltanschauliche Minderheiten nicht ausgrenzt, sie verlangt vielmehr auch, dass diese sich selbst nicht abgrenzen und sich einem Dialog mit Andersdenkenden und -gläubigen nicht verschließen. Für eine offene pluralistische Gesellschaft bedeutet der Dialog mit solchen Minderheiten eine Bereicherung. Dies im Sinne gelebter Toleranz einzuüben und zu praktizieren, ist wichtige Aufgabe schon der Grundschule. Das Vorhandensein eines breiten Spektrums von Überzeugungen in einer Klassengemeinschaft kann die Fähigkeit aller Schüler zu Toleranz und Dialog als einer Grundvoraussetzung demokratischer Willensbildungsprozesse nachhaltig fördern" (BVerfG, a.a.O.).

Unter Verweis auf diese Zielsetzung wird nicht nur das Homeschooling in ständiger Rechtsprechung unterbunden, sondern werden auch Anträge auf punktuelle Unterrichtsbefreiung versagt. Berühmt ist die Krabat-Entscheidung des Bundesverwaltungsgerichts aus dem Jahre 2013. Es ging um die Frage, ob ein Siebtklässler, der wie seine Eltern den Zeugen Jehovas angehörte, an der schulischen Vorführung einer Verfilmung von Otfried Preußlers Krabat teilzunehmen habe. Die Familie hatte die Befreiung für die Dauer des Filmes aus religiösen Gründen beantragt, weil im Film „schwarze Magie“ zu sehen sei. Das Bundesverwaltungsgericht lehnte dies letztinstanzlich ab und stützte sich dafür auf die „Integrationsfunktion“ der Schule. Sie folge

„einer starren, gleichwohl aber verfassungsrechtlich tragfähigen Modellvorstellung: Der einzelne Schüler soll an sämtlichen schulischen Veranstaltungen teilnehmen müssen, weil nur die permanente, obligatorische Teilhabe am Schulunterricht unter Hintanstellung aller entgegenstehenden individuellen Präferenzen gleich welcher Art jenen gemeinschaftstiftenden Effekt zu erzeugen vermag, der mit der Schule bezweckt wird und der die Einführung der staatlichen Schulpflicht zu wesentlichen Anteilen legitimiert" (Bundesverwaltungsgericht, Urteil vom 11.9.2013 - 6 C 12/12, abgedruckt in: Neue Juristische Wochenschrift 2014, S. $804 \mathrm{ff}$.).

Die Statuierung einer schulischen Integrationsaufgabe, die neben den Bildungs- und Erziehungsauftrag tritt, hat weitreichende Bedeutung im Kleinen wie im Großen: für individuelle Befreiungsanträge wie für die Gestaltung des Schulwesens insgesamt (Avenarius, 2015; krit. Wiezorek \& Reimer, 2021).

Dabei sind zwei gegenläufige Entwicklungen zu beobachten: Die juristische Literatur sieht und thematisiert die Schulpflicht zunehmend in ihrer Intensität als recht- 
fertigungsbedürftigen Grundrechtseingriff (vorbildlich Rux, 2018, S. $39 \mathrm{ff}$.); ferner beginnt sie sich für das Phänomen des Homeschoolings zu interessieren (Handschell, 2012; Hauk, 2020; Reimer, 2012; von Lucius, 2017). Auch kommt allmählich das „Freilernen“ in den Blick (Rux, 2018, S. 50 f., 104), jenes Lernmodell also, das vom Homeschooling konzeptionell und soziologisch - vom Anspruch wie vom Milieu her - deutlich zu unterscheiden ist, insofern es Beschulung auch im häuslichen Kontext ablehnt, um stattdessen auf das selbstbestimmte und eigenverantwortliche Lernen des Kindes zu setzen (Edel, 2016; Kern, 2016).

Demgegenüber scheinen Schulbehörden und Gerichte ihren Kurs der Ablehnung von Befreiungsanträgen und der Sanktionierung von Familien, die Homeschooling oder Freilernen betreiben, eher noch zu verschärfen. So kam es in Bundesländern, in denen entsprechende Straftatbestände bestehen, tatsächlich zu strafgerichtlichen Verurteilungen von Eltern, die mit ihren Kindern Homeschooling praktizierten. Das Bundesverfassungsgericht hält dies für grundgesetzkonform (2. Kammer des Zweiten Senats, Beschluss vom 15.10.2014 - 2 BvR 920/14, abgedruckt in: Neue Juristische Wochenschrift 2015, S. $44 \mathrm{ff}$.).

\subsection{Deutsche Schulpflicht und Europäische Menschenrechtskonvention}

Die Nichtzulassung von Homeschooling und Freilernen in Deutschland stellt im europäischen Vergleich eine Anomalie dar. In den meisten europäischen Staaten sind diese Formen schulfernen Lernens zulässig. Die Vermutung liegt nahe, dass die deutsche Praxis eine Grenze in der Europäischen Menschenrechtskonvention (EMRK) finden könne. Die EMRK enthält als einschlägige Verbürgungen das Recht auf Achtung des Privat- und Familienlebens (Art. 8), die Gedanken-, Gewissens- und Religionsfreiheit (Art. 11) und das Recht auf Bildung (Art. 2 des ersten Zusatzprotokolls). Danach darf niemandem

„das Recht auf Bildung verwehrt werden. Der Staat hat bei Ausübung der von ihm auf dem Gebiet der Erziehung und des Unterrichts übernommenen Aufgaben das Recht der Eltern zu achten, die Erziehung und den Unterricht entsprechend ihren eigenen religiösen und weltanschaulichen Überzeugungen sicherzustellen“ (ebd.).

Dennoch wird bislang auch auf der Ebene der Europäischen Menschenrechtskonvention die deutsche Praxis strikter Unterbindung von Homeschooling und Freilernen unbeanstandet gelassen. So hat der Europäische Gerichtshof für Menschenrechte in Straßburg die durch deutsche Familiengerichte angeordnete Entziehung des Sorgerechts heimbeschulender Eltern (vgl. hierzu näher Vogt, in diesem Band S. 451-460) in einem hessischen Fall für mit der Europäischen Menschenrechtskonvention vereinbar gehalten (Wunderlich gg. Deutschland, Urteil vom 10.01.2019, Kammer V, Be- 
schwerde-Nr. 18.925/15). Entscheidendes Argument war - wie häufig - der Ermessensspielraum der Vertragsstaaten („margin of appreciation“). Aber auch insoweit finden sich in der Literatur abwägende Ansätze. So gewähre die EMRK grundsätzlich keinen Anspruch auf Hausunterricht.

„In eng begrenzten Ausnahmefällen, in denen die Persönlichkeit der Eltern oder gar die der Kinder am Konflikt zu zerbrechen droht, kann allerdings die Duldung von Hausunterricht geboten sein. Der Wegzug aus Deutschland ist hier weder menschenrechtlich noch rechtspolitisch eine Alternative" (Marauhn, 2012, S. 108).

Anders als der Europäische Gerichtshof für Menschenrechte (als Organ des Europarats) hat sich der Europäische Gerichtshof in Luxemburg (als Organ der Europäischen Union), soweit ersichtlich, noch nicht mit den Themen Homeschooling und Freilernen befasst.

\section{Die Schulpflicht in der Pandemie}

Was die Corona-Pandemie in schulrechtlicher Hinsicht verändert oder aber ans Licht gebracht hat, lässt sich derzeit noch nicht überblicken (verfassungsrechtliche Bewertung des ersten Lockdowns bei Pernice-Warnke \& Warnke, 2020; eingehend Lohse, 2021). Mit Gewissheit kann man bislang nur sagen, dass die Pandemie auch im Schulverfassungsrecht zahlreiche Gewissheiten erschüttert hat. Die folgenden Passagen greifen die Frage auf, was man über die Schulpflicht aus den Erfahrungen der Pandemie lernen kann. Ist sie suspendiert worden, oder hat sie lediglich eine andere Form angenommen, sodass man für die Zukunft die Schulpflicht auch als Bildungspflicht konzeptualisieren könnte?

\subsection{Vom Präsenzunterricht zum Distanzunterricht und zurück}

$\mathrm{Zu}$ Beginn der Pandemie schien aus der Schulpflicht, verstanden als Schulbesuchspflicht, über Nacht ein Schulbesuchsverbot in Verbindung mit einem Homeschoolinggebot zu werden. Instrumente für den Übergang aus dem gewohnten Zustand in die neue Phase des kollektiven Homeschoolings (d.h. eines verpflichtenden Distanzlernens) waren nicht primär schulrechtliche, sondern infektionsschutzrechtliche Maßnahmen, insbesondere die auf $\$ 32$ in Verbindung mit $\$ 28$ Infektionsschutzgesetz (IfSG) gestützten Rechtsverordnungen der Landesregierungen, in geringerem Umfang auch Allgemeinverfügungen (Lohse, 2021, S. 69 f., 77 f.). Was die Schulen anging, so wurde der Lockdown im März 2020 eingeleitet durch Formulierungen wie: 
„Es wird allgemein angeordnet, dass Schülerinnen und Schüler dem Unterricht und anderen regulären schulischen Veranstaltungen an Einrichtungen gem. $\$ 33$ Nr. 3 des Infektionsschutzgesetzes bis zum 19. April 2020 fernbleiben müssen. Ihr Fehlen gilt als entschuldigt. Satz 1 und 2 gelten nicht für die Abnahme von Prüfungsleistungen“" ( $\$ 3$ der Zweiten [hessischen] Verordnung zur Bekämpfung des Corona-Virus vom 13.03.2020, GVBl. S. 153).

Obwohl den Schulgemeinschaften (Lehrenden, Lernenden und Eltern) am prominentesten die Elternbriefe der Kultusministerinnen und Kultusminister entgegentraten, so waren diese doch nicht die juristischen Steuerungsinstrumente, sondern nur Kommunikationsmittel. Auch Erlasse und Empfehlungen der Kultusministerinnen und Kultusminister spielten, rechtlich gesehen, keine zentrale Rolle, ebenso wenig wie die Schulgesetze, die nur vereinzelt geändert wurden (Lohse, 2021, S. 70). Es war vielmehr das Infektionsschutzrecht, das - vor allem durch Rechtsverordnungen der Landesregierungen - dem Bildungswesen seinen neuen Rahmen gab. Dieser tiefe Eingriff in das Schulrecht wurde kompetenziell (im Bund-Länder-Verhältnis) dadurch abgemildert, dass mit den Infektionsschutzverordnungen das Hauptinstrument zur Bekämpfung der Pandemie in den Händen der Länder lag. Und so wunderlich es aussehen mag, dass eine bislang verfassungsunmittelbar begründete Pflicht - Schulpflicht, strikt als Schulbesuchspflicht verstanden - durch einfache Rechtsverordnung soll ausgesetzt werden können, so tragfähig mag dies doch verfassungsrechtlich sein (a.A. wohl Lohse, 2021, S. 79). Denn das Infektionsschutzgesetz als kompetenzgerechte bundesrechtliche Norm (Art. 74 Abs. 1 Nr. 19 GG) kann zu Infektionsschutzzwecken auch schulbezogene Regelungen treffen. Zwar konterkarieren oder modifizieren diese Regelungen - auf Zeit - Verfassungsgüter wie den staatlichen Bildungs- und Erziehungsauftrag (von Rechtsprechung und herrschender Meinung in der Literatur konkretisiert zu einer Schulbesuchspflicht, s. o., Kap. 2.2) oder ein Recht auf Bildung der Schülerinnen und Schüler; doch kann dies verfassungsrechtlich durch die staatliche Schutzpflicht für Leib und Leben (Art. 2 Abs. 2 Satz 1 GG) gerechtfertigt sein. Dies setzt selbstverständlich voraus, dass eine sorgfältige Abwägung getroffen wird und diese ergibt, dass der infektionsschutzrechtliche Eingriff verhältnismäßig ist (für den ersten Lockdown bejaht von Pernice-Warnke \& Warnke, 2020). Dabei sind die stark asymmetrischen Folgen des Verbots eines Schulbesuchs in Rechnung zu stellen, d.h. die Tatsache, dass je nach familiärem Hintergrund einige Kinder besonders nachteilig betroffen sind. Insofern ist Chancengleichheit nicht nur ein politisches, sondern auch ein verfassungsrechtliches Argument.

Was den Wechsel zwischen den Modellen angeht, so behalten die Landesregierungen ihr Flexibilitätsreservoir: So schnell, wie aus der Präsenzschulpflicht eine Distanzlernpflicht geworden ist, kann dieser Prozess durch Aufhebung oder Änderung der infektionsschutzrechtlichen Rechtsverordnungen auch wieder umgekehrt werden, ohne dass auch nur ein Iota der Schulgesetze (oder gar der Verfassungen) geändert werden müsste. 


\subsection{Distanzlernpflicht?}

Die eigentliche Frage aber lautet, ob ein Betretungsverbot (wie das oben abgedruckte hessische) oder Schulschließungen (durch Allgemeinverfügung oder Rechtsverordnung) die Schulbesuchspflicht in eine Pflicht zur häuslichen Unterrichtsteilnahme umwandeln kann. Nach einer Auffassung bleibt die gesetzlich angeordnete Schulbesuchspflicht bestehen; sie könne nicht durch untergesetzliche Normen oder gar Allgemeinverfügungen landesweit ausgesetzt werden (Lohse, 2021, S. 79 f.). Vielmehr müssten Regelungen getroffen werden, die einerseits die Präsenzpflicht aussetzen, andererseits festlegen, ob und wie die Schulbesuchspflicht und der Unterrichtsanspruch durch Distanzunterricht erfüllt werden können (ebd., S. 80). Diese Regelungen oblägen den Kultusministerien der Länder, da sie nicht mehr zu den Infektionsschutzmaßnahmen nach $\$ \$ 28$, 28a IfSG gezählt werden könnten (ebd., a. a. O.). Dem lässt sich entgegenhalten, dass die Schulbesuchspflicht aus infektionsschutzrechtlichen Gründen suspendiert werden kann, dies die Schulpflicht aber gar nicht berührt. Insofern lässt sich überzeugender von einem „Fortbestehen der Schulpflicht bei Aufhebung der Präsenzpflicht gekoppelt mit einem Betretungs- und Präsenzunterrichtsver-

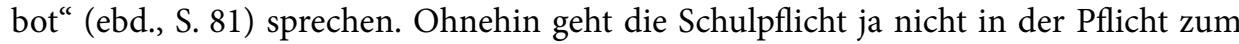
Schulbesuch auf. Die Schulpflicht ernst zu nehmen bedeutet in bestimmten Situationen gerade, den Schulbesuch zu verbieten. Sie entpuppt sich als eine Bildungspflicht, die in der Regel, aber eben nicht stets und ständig, erst recht nicht begrifflich notwendig eine Schulbesuchspflicht enthält.

\subsection{Schulpflicht als Bildungspflicht}

Wenn sich in der Pandemie wie in einem Brennglas Schwächen der Gesellschaft und ihrer Systeme zeigen, dann wohl in besonderer Weise hier. Denn die bisherige Rechtsprechung und Lehre sind weit überwiegend nicht davor zurückgeschreckt, Schule in Anlehnung an einen von Hans Heckel 1955 geprägten Schulbegriff zu definieren als

„eine auf gewisse Dauer berechnete, an fester Stätte unabhängig vom Wechsel der Lehrer und Schüler in überlieferten Formen organisierte Einrichtung der Erziehung und des Unterrichts, die durch planmäßige und methodische Unterweisung eines größeren Personenkreises in einer Mehrzahl allgemeinbildender oder berufsbildender Fächer bestimmte Bildungs- und Erziehungsziele zu verwirklichen bestrebt ist, und die nach Sprachsinn und allgemeiner Auffassung als Schule angesehen wird" (Heckel, 1955, S. 218; Nachweise zur anhaltenden Bezugnahme auf die Formel bei Reimer, 2021, S. 62 f.).

Schulpflicht ist dann im Kern tatsächlich Schul(haus)besuchspflicht. Ob dies dem Sinn des Bildungsverfassungsrechts gerecht wird, erscheint zweifelhaft. Hier könnte gleich auf zwei Ebenen die Verwechslung von Zweck und Mittel zugrunde liegen: 
Denn verfassungsrechtlich scheint das Telos der Schulpflicht die Bildung und Erziehung (ggf. auch die Integration) der jungen Menschen zu sein; ihnen dient die Schule. Der Schule wiederum dient das gemeinsame Lernen im Schulhaus. Der Besuch der „festen Stätte“ ist Instrument für das schulische Lernen, schulisches Lernen Instrument für den staatlichen Bildungs- und Erziehungsauftrag. Im Regelfall sind diese Instrumente sachangemessen und funktional; in Grenzfällen (sei es auf individueller, sei es auf gesellschaftlicher Ebene) unangemessen und dysfunktional. Dann darf das Instrument keinen Selbststand erhalten, weil es seinem Zweck nicht mehr dient. In diesem Sinne ist in jüngerer Zeit dem traditionellen Schulbegriff ein funktionaler Schulbegriff gegenübergestellt worden (Brosius-Gersdorf, 2013, S. 959 ff.): Danach ist als Schule

„jede Form der Vermittlung von Bildung und bildungsbezogener Erziehung zu verstehen, die geeignet und erforderlich ist, um Kinder zu selbstbestimmten und verantwortungsbewussten, in die soziale Gemeinschaft integrierten Bürgern zu entwickeln und ihre Teilhabe an Ausbildung, Studium und Beruf sicherzustellen“" (Brosius-Gersdorf, 2013, S. 960).

Ob man so weit gehen und damit den Schulbegriff weitgehend dekonturieren muss, mag an dieser Stelle offenbleiben (vgl. Reimer, 2021, S. 68 ff.). Was sich jedenfalls in der Pandemie im Großen gezeigt hat - dass Schulpflicht in ihrem Sinnkern Bildungspflicht ist -, muss auch in anderen Situationen beherzigt werden, und zwar auch im (scheinbar) Kleinen, auf der Ebene des einzelnen Kindes oder der einzelnen Familie. Wenn sich etwa für eine Schülerin oder einen Schüler die Verwirklichung der Bildungs- und Schulpflicht durch Schulbesuchspflicht als dysfunktional erweist, weil die Persönlichkeit daran zerbricht (zum Kriterium: Marauhn, 2012, S. 108), dann kann sich die Schulbesuchspflicht aus verfassungsrechtlichen Gründen in eine andere Form der Schulpflicht wandeln, beispielsweise in die Pflicht, sich nach den Curricula der Schule an einem anderen Ort zu bilden. Sonst wird die allgemeine Schulpflicht entgegen ihrem Ziel, Bildungsgleichheit zu eröffnen, zu einem Mittel, Bildungsungleichheit zu erzwingen.

\section{Perspektiven}

Schulpflicht lässt sich nach hier vertretener Auffassung nicht mehr überzeugend als Schulbesuchspflicht definieren. Sie muss vielmehr ihre anstaltlichen ,Eierschalen' abwerfen und sich als (bedingte) Bildungspflicht verstehen, die in aller Regel durch Schule und - konkreter - durch Schulbesuch zu verwirklichen ist, aber weder begrifflich noch in jedem Einzelfall teleologisch mit dem Schulbesuch steht und fällt. Im Gegenteil kann der staatliche Bildungs- und Erziehungsauftrag (der richtigerweise nicht in Art. 7 Abs. 1 GG, sondern in den schulbezogenen und grundrechtlichen Vorschriften des Landesverfassungsrechts verankert zu sehen ist) im Einzelfall oder 
gar in der Breite - wie die Pandemie gelehrt hat - den Verzicht auf Schulbesuch, ja sogar das Verbot des Schulbesuchs gebieten. Die Pflicht, sich im Rahmen des verfassungsrechtlichen Bildungs- und Erziehungsauftrags zu bilden, besteht fort. Schule ist das wertvollste Instrument des staatlichen Bildungs- und Erziehungsauftrags und der Verwirklichung bildungsbezogener Chancengleichheit, aber eben nur ein Instrument: Mittel, nicht Zweck.

\section{Literatur und Internetquellen}

Hinweis der Redaktion: Als Hilfestellung sind die wesentlichen rechtlichen Regelungen, auf die in den Beiträgen des Heftes Bezug genommen wird, in einem das Heft begleitenden Online-Anhang abgedruckt. Er ist unter folgendem Link abrufbar: https://www.waxmann.com/ artikelART104608

Anschütz, G. (1933). Die Verfassung des Deutschen Reichs vom 11. August 1919. Ein Kommentar für Wissenschaft und Praxis (14. Aufl., Nachdruck 1965). Darmstadt: WBG.

Avenarius, H. (2015). Schulpflicht vs. Homeschooling. Die neuere Rechtsprechung des BVerfG zur Integrationsaufgabe der öffentlichen Schule. Neue Zeitschrift für Familienrecht, 2 (8), 342-346.

Avenarius, H., \& Hanschmann, F. (2019). Schulrecht. Ein Handbuch für Praxis, Rechtsprechung und Wissenschaft (9. Aufl.). Köln: Carl Link.

Brosius-Gersdorf, F. (2013). Kommentierung von Art. 7 GG. In H. Dreier (Hrsg.), Grundgesetz. Kommentar. Bd. 1 (3. Aufl.). Tübingen: Mohr Siebeck.

Edel, J. (2016). Schulfreie Bildung. Über die Vernachlässigung schulfreier Bildungskonzepte in Deutschland. Leipzig: Tologo.

Handschell, T. (2012). Die Schulpflicht vor dem Grundgesetz. Geschichte der Schulpflicht und ihre verfassungsrechtliche Bewertung vor dem Hintergrund des sogenannten Homeschooling (Studien zum Schul- und Bildungsrecht, Bd. 3). Baden-Baden: Nomos. https://doi. org/10.5771/9783845241333

Hanschmann, F. (2017). Staatliche Bildung und Erziehung. Ganztagsschule, Bildungsstandards und selbständige Schule als Herausforderungen für das Verfassungs- und Schulrecht. Tübingen: Mohr Siebeck. https://doi.org/10.1628/978-3-16-154879-6

Hauk, J. (2020). Die Pflicht zum Schulbesuch. Zur Frage nach einem Recht auf Homeschooling unter Berücksichtigung grundrechtlicher Freiheiten von Eltern und Schülern. Baden-Baden: Nomos.

Heckel, H. (1955). Deutsches Privatschulrecht. Berlin, Köln: Heymann.

Huster, St. (2014). Endlich: Abschichtung statt Abwägung. Die Öffentliche Verwaltung, 67 (20), 860-866.

Kern, M. (Hrsg.). (2016). Selbstbestimmte und selbstorganisierte Bildung versus Schulpflicht. Betrachtungen zum Spannungsverhältnis zwischen Schulbesuchspflicht und den Grundrechten der jungen Menschen. Leipzig: Tologo.

Lohse, E. J. (2021). \$2 Schulische Bildung. In Ch. Birnbaum (Hrsg.), COVID-19. Bildungsrecht in der Corona-Krise. Frühkindliche Bildung - Schule - Hochschule - Berufsbildung (S. 45-128). München: C. H. Beck.

Marauhn, T. (2012). Hausunterricht zwischen Bildungsrecht und Elternrecht. „Homeschooling" im Lichte der EMRK. In F. Reimer (Hrsg.), Homeschooling. Bedrohung oder Bewährung des freiheitlichen Rechtsstaats (S. 99-108). Baden-Baden: Nomos. https://doi. org/10.5771/9783845237367-99 
Mors, A. (1986). Die Entwicklung der Schulpflicht in Deutschland. Tübingen: Diss.

Pernice-Warnke, S., \& Warnke, C. (2020). Bildungseinrichtungen in der SARS-CoV-2-Pandemie. Die öffentliche Verwaltung, 1089-1100.

Reimer, F. (2008). Allgemeine, nicht absolute Schulpflicht. In J. P. Thurn \& F. Reimer (Hrsg.), Homeschooling als Option? Neue Zeitschrift für Verwaltungsrecht, 7, 718-722.

Reimer, F. (Hrsg.) (2012). Homeschooling. Bedrohung oder Bewährung des freiheitlichen Rechtsstaats (Schriften zum Bildungs- und Wissenschaftsrecht, Bd. 13). Baden-Baden: Nomos. https://doi.org/10.5771/9783845237367

Reimer, F. (2021). Der verfassungsrechtliche Schulbegriff: Neuer Wein in neue Schläuche! Recht der Jugend und des Bildungswesens, 69 (1), 58-77.

Rux, J. (2018). Schulrecht (6. Aufl.). München: C. H. Beck.

von Lucius, J. (2017). Homeschooling: Eine verfassungsrechtliche Untersuchung des Rechts der Eltern, ihre Kinder zu Hause zu unterrichten (Gesetzgebung und Verfassung, Bd. 9). Baden-Baden: Nomos. https://doi.org/10.5771/9783845272252

Wiezorek, C., \& Reimer, F. (2021). Der Integrationsauftrag der Schule - Erziehungs- und rechtswissenschaftliche Perspektiven. Erziehungswissenschaft, 32 (62), 23-33. https:// doi.org/10.3224/ezw.v32i1.03

Wißmann, H. (2012). Das allgemeine Schulwesen: Projekt der Moderne - Programm der Freiheit? In F. Reimer (Hrsg.), Homeschooling. Bedrohung oder Bewährung des freiheitlichen Rechtsstaats (S. 17-54). Baden-Baden: Nomos. https://doi.org/10.5771/ 9783845237367-17

Franz Reimer, Prof. Dr., geb. 1971, Inhaber der Professur für Öffentliches Recht und Rechtstheorie an der Justus-Liebig-Universität Gießen.

E-Mail: franz.reimer@recht.uni-giessen.de

Korrespondenzanschrift: Justus-Liebig-Universität Gießen, Professur für Öffentliches Recht und Rechtstheorie, Hein-Heckroth-Str. 5, 35390 Gießen 